\section{SCIDOC \\ Publishers}

\title{
Coupled Chemical Reactions in Dynamic Nanometric Confinement: VI. Neutron Depth Profiling Studies of Nanofluidic Behaviour During Track Etching in Polymers
}

Research Article

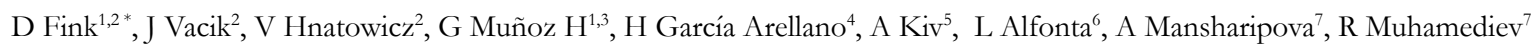

${ }^{1}$ Division de Ciencias Naturales e Ingineria, Universidad Autónoma Metropolitana-Cuajimalpa, Pedro Antonio de los Santos Col. Sn. Miguel Chapultepec, D.F. México

${ }^{2}$ Nuclear Physics Institute, ASCR, Řež, Czech Republic

${ }^{3}$ Departamento de Fisica, Universidad Autónoma Metropolitana-Iztapalapa, D.F., México

${ }^{4}$ Departamento de Ciencias Ambientales, División de Ciencias Biológicas y de la Salud, Universidad Autónoma Metropolitana-Lerma, Av. de las Garzas Col. El Panteón, Lerma de Villada, Municipio de Lerma, Estado de México.

${ }^{5}$ Department of Materials Engineering, Ben-Gurion University of the Negev, Beer-Sheva, Israel.

${ }^{6}$ Avram and Stella Goldstein-Goren Department of Biotechnology Engineering, Ben-Gurion University of the Negev, Beer-Sheva, Israel.

${ }^{7}$ Almaty University, Kazakhstan.

\section{Abstract}

In recent papers it was shown (by current/voltage (I/V) recordings, Bode plots and Ion Transmission Spectrometry) that the formation of precipitates from solutions in swift heavy ion tracks in thin polymer foils, if coupled with polymer foil etching, eventually leads to the formation of membranes within the etched tracks. Such structures open promising perspectives for the creation of novel biosensors. In this paper we report on Neutron Depth Profiling (NDP) studies of samples where such coupled chemical reactions were initiated in the presence of $\mathrm{Li}^{+}$and $\mathrm{F}^{-}$ions. Whereas $\mathrm{I} / \mathrm{V}$ spectroscopy gave us clues about the different migration speeds of both $\mathrm{Li}^{+}$and $\mathrm{F}$ - ions in latent and etched tracks, NDP revealed that $\mathrm{Li}$ was always most abundant at the etched track entrances, especially on the track side where the precipitation-forming ions are applied.

\section{Introduction}

The coupling of two chemical-topological reactions with each other was a main topic of recent research, as in this way membranes of $\mathrm{Ag}_{2} \mathrm{O}$ or $\mathrm{LiF}$ can be formed within etched ion tracks, thus separating them into two adjacent segments. Such structures open promising perspectives for the creation of novel biosensors, when cladding the etched track walls with e.g., enzymes. If measuring simultaneously the same biomolecule specie in both segments, the Coulombic interaction of the enzymatic reaction products in both segments leads to polarization effects across the membranes that improve the sensor efficiency (to be published). Also, such segmented sensors may act as "electrostatic bottles" to accumulate enzymatic reaction products during a certain measuring interval, thus enhancing the biosensor's registration threshold (to be published).
Often, coupled chemical-topological reactions (CCR) consist of two subsequent stages: A) the pre-CCR stage: rapid preferential dissolution of the polymer along the radiation-damaged zones, i.e. the transformation of the so-called "latent tracks" (= asirradiated regions) to "etched tracks" (= irradiated regions after chemical removal of their radiochemical products), and B) the (usually slow) precipitation-forming (i.e., membrane-forming) CCR stage within the ion tracks.

Two principle cases have to be distinguished, one where the ions A required for etching of the latent tracks react with the precipitation-forming $\mathrm{B}$ ions, and one where they do not react. Whereas in the second case, the membrane-forming ions B can coexist with the etchant solution A due to their inertness, this is not possible in the first case. Here both the pure track etching reaction A

\footnotetext{
*Corresponding Author:

Dietmar Fink,

Division de Ciencias Naturales e Ingineria, Universidad Autónoma Metropolitana-Cuajimalpa, Pedro Antonio de los Santos 84, Col. Sn. Miguel Chapultepec, C.P.11850, México, D.F.; México.

Tel/Fax: 00493080496233

E-mail: fink@xanum.uam.mx

Received: October 26, 2016

Accepted: November 22, 2016

Published: November 24, 2016

Citation: D Fink, J Vacik, V Hnatowicz, G Muñoz H, H García Arellano, et al., (2016) Coupled Chemical Reactions in Dynamic Nanometric Confinement: VI. Neutron Depth Profiling Studies of Nanofluidic Behaviour During Track Etching in Polymers. Int J Bioanal Methods Bioequival Stud. 3(2), 55-61. doi: http://dx.doi.org/10.19070/2470-4490-160007

Copyright: D Fink ${ }^{\circ}$ 2016. This is an open-access article distributed under the terms of the Creative Commons Attribution License, which permits unrestricted use, distribution and reproduction in any medium, provided the original author and source are credited.
} 
and the precipitation-forming reaction $\mathrm{B}$ have to be decoupled by performing them sequentially. Whereas we had presented previously the reaction: $2 \mathrm{Ag}^{+}+2 \mathrm{OH}^{-} \rightarrow 2 \mathrm{AgOH} \downarrow \rightarrow \mathrm{Ag}_{2} \mathrm{O} \downarrow+\mathrm{H}_{2} \mathrm{O}$ \{with $\mathrm{Ag}^{+}$being the precipitate-type (B) ion and $\mathrm{OH}^{-}$being the etching-type ion (A) $\}[1,2]$ as an example for the second case, we chose the precipitate-type reaction $(\mathrm{B}): \mathrm{Li}^{+}+\mathrm{F}^{-} \rightarrow \mathrm{LiF} \downarrow$ and the etching-type reaction $(\mathrm{A}): \mathrm{OH}^{-}+$polymer $\rightarrow$ \{soluble polymer reaction products $\}$, as an example for the first case [3].

For convenience, we denote in this paper the side of the precipitation-forming ions as the "Cis" side" (in the NDP profiles shown in this work, this is always the left side). The side where the etchant ions are located during this third step (i.e., the right side of the NDP profiles) will be always called the "Trans-side".

The membrane formation within the etched tracks by the CCR strategy is by far not as simple as we originally thought. The basic precondition for the formation of impermeable membranes is the firm anchoring of the precipitates (e.g., LiF) on the ion track walls from the very moment of breakthrough on. If during the subsequent continuing etching the track radii in the contact region of both track segments increase, leaks will occasionally occur between the membrane rim and the etched track walls that promote new CCR until these leaks are "repaired" by blocking by the new precipitations. This means, radial membrane growth will occur in distinct steps, with "quiet phases" in between, during which the membranes keep both track compartments hermetically sealed from each other and hence also suppress any passing electrical current (if a constant or low-frequency voltage is applied across the membrane). These "quiet phases" were therefore considered to be the characteristic fingerprints of membrane formation (for examples, please consult the figures in Refs. [1-3]).

If the formed membranes are not stable, they break and their fragments are swept out from the etched tracks due to the osmotic pressure difference induced by both the different liquids on both foil sides, and eventually also by the test voltage applied across the foil. The same happens if the reacting chemical compounds (here: $\mathrm{Li}$ and $\mathrm{F}$ salt solutions) are inserted into already fully transparent nanopores: then the tracks just act as nanoparticle generators for the precipitations, as the latter have no possibility to anchor and form membranes. In both cases we encounter a situation which is dominated by nanofluidic behaviour [4]:

Negative stationary charges on the etched track walls (arising from dangling bonds created by the etchant) attract the cations of the inserted liquids (here: $\mathrm{H}^{+}, \mathrm{Li}^{+}$) within the Debye layer, whereas the corresponding anionic counter-ions (here: $\mathrm{OH}^{-}$and $\mathrm{F}^{-}$) can move only at distances larger than the Debye layer, provided that the etched track radii exceed the thickness of the electrical double layer (ELD); otherwise the counter-ion transport is fully blocked). Therefore (and also taking into account that nanofluidic transport is purely laminar), application of an external electric field along the track direction makes both counter-ions pass smoothly each other into opposite directions without any interaction. Only at the track exits (or within $\mu \mathrm{m}$-large etched tracks) the laminar flow turns towards turbulent, and thorough intermixing - the basic condition for any efficient chemical reaction - with precipitation formation takes place. Due to the shielding of the etched track walls by the electric double layer, the precipitations cannot anchor there and are therefore swept out.
This means, whereas permanent nanoparticle generation and ejection is the normal behavior of etched tracks embedded in between two precipitation-forming liquids, the generation of stable membranes within the tracks is expected to occur essentially during (and very shortly after) the very moment of etchant breakthrough. Prolonged etching will turn the track population from tracks with membranes to a population of tracks completely filled with precipitations. Whereas in previous works, these mechanism were studied by recording of the electrical properties (current/ voltage studies, determination of four-pole parameters, Bode plots, Fourier spectroscopy) [1-3] and by ion transmission spectrometry (ITS) [5], we concentrate in this work on the findings obtained by neutron depth profiling (NDP) [6]. Here, the concentration distribution of the $\mathrm{LiF}$ reaction products were measured along the whole lengths of the ion tracks.

\section{Experimental}

Sample preparation: for these examinations, we took $12 \mu \mathrm{m}$ thick mylar (PET) foils which were irradiated at JINR Dubna with $250 \mathrm{MeV} \mathrm{Kr}$ ions up to a fluence of $4 \times 10^{6} \mathrm{~cm}^{-2}$. (samples \#123, \#119), $80 \mathrm{MeV} \mathrm{Kr}$ ions up to a fluence of $4.5 \times 10^{6} \mathrm{~cm}^{-2}$ (\#122), and $80 \mathrm{MeV} \mathrm{Kr}$ ions up to a fluence of $5 \times 10^{7} \mathrm{~cm}^{-2}$ (\#120). Thereafter they were cut to $\sim 1 \mathrm{~cm}^{2}$ large pieces and inserted into the center of a specially constructed experimental chamber so that the right and left compartments of that chamber were hermetically sealed from each other by that foil. $\mathrm{Ag} / \mathrm{AgCl}$ electrodes were inserted into both these compartments and a sinusoidal alternating voltage $\mathrm{U}$ between $\sim 1$ and 10 Vpeak-peak at $\sim 0.5 \mathrm{~Hz}$ from a Velleman PCSGU250 pulse generator/oscilloscope combination was applied through them to the system for recording the protocol of the reaction kinetics. Alternating voltages were applied to detect eventual phase shifts between voltage and transmitted current (to obtain information about the type of conductivity) and possible track rectification (to obtain information about track surface charges and asymmetries of their distributions). The emerging current through the PET foil between both compartments was constantly monitored, to obtain a protocol of the foil manipulation procedures.

As $\mathrm{Li}^{+}$ions do not interact with the $\mathrm{NaOH}$ etchant by precipitation, two experimental strategies are possible: one can either perform the etching and the precipitation steps of the CCR strategy separately, one after each other ("sequential approach"), or simultaneously ("simultaneous approach"). Here we compare both approaches with one another. Table 1 compiles the experimental conditions.

The well known NDP technique [6] was used for depth profiling of $\mathrm{Li}$ via the ${ }^{6} \mathrm{Li}(\mathrm{n}, \alpha)^{3} \mathrm{H}$ reaction. The measurements were accomplished on the NDP device (CANAM infrastructure) installed at the horizontal channel of LWR-15 research reactor at NPI (NRI Research center operated by CV Rež). The samples were irradiated with thermal neutrons from a $6 \mathrm{~m}$ long neutron guide $\left(10^{7} \mathrm{~cm}^{-2} \mathrm{~s}^{-1}\right)$ and the reaction products were registered by a surface barrier detector connected to a standard spectrometric chain. Due to the very low amount of $\mathrm{Li}$ in our etched tracks during the breakthrough process, and due to the necessity to have a sufficiently high statistics of the NDP measurement, the measurements typically required a few weeks per sample. Furthermore, the measurements had to be performed from both foil sides, to 
Table 1. Systematic overview about the LiF cases measured in this work, and comparison with previous results based on $\mathrm{I} / \mathrm{U}$ results only. $\mathrm{t}_{\mathrm{CCR}}$ is the time after which the I/U recording stopped so that both the ITS and NDP measurements could take place. $\mathrm{T}=$ etching temperature. All applied voltages were of sinusoidal shape with $\sim 0.5 \mathrm{~Hz}$. The Cis-side corresponds to the left side of the NDP spectra and the Trans-side to the right NDP spectrum side. The foil numbers refer to the protocol underlying the experiments in the previous publication [2].

\begin{tabular}{|c|c|c|c|c|c|}
\hline $\begin{array}{l}\text { Fig., } \\
\text { foil \# }\end{array}$ & I/U Observations & $\begin{array}{c}\text { Electrolytes, } \\
\text { Cis-Side }\end{array}$ & $\begin{array}{c}\text { Electrolytes, } \\
\text { Trans-Side }\end{array}$ & $\begin{array}{r}t_{\mathrm{CCR}} \\
\text { Min } \\
\end{array}$ & $\mathbf{T}^{\circ} \mathrm{C}$ \\
\hline \multirow[b]{2}{*}{1 \#123 } & \multirow{2}{*}{$\begin{array}{l}\text { Sequential approach: } \\
\text { First etching from both sides without } \mathrm{Li}^{+} \text {and } \mathrm{F}^{-} \text {pres- } \\
\text { ence, etching stopped before breakthrough; thereafter } \\
\text { continuation of etching from Trans side only with } \\
\mathrm{NaOH} / \mathrm{NaF} \text { solution, whereas Cis side is filled with } \mathrm{LiCl} \\
\text { solution only: Near-Ohmic current, possibly transient } \\
\mathrm{LiF} \text { membrane formation }\end{array}$} & $\begin{array}{c}\text { First: } \\
9 \mathrm{M} \mathrm{NaOH}\end{array}$ & $\begin{array}{c}\text { First: } \\
9 \mathrm{M} \mathrm{NaOH}\end{array}$ & 12 & 25 \\
\hline & & $\begin{array}{l}\text { Second: } \\
10 \mathrm{M} \mathrm{LiCl}\end{array}$ & $\begin{array}{l}\text { Second: } \\
\sim 50 \mathrm{vol} \%(1 \\
\mathrm{M} \mathrm{NaF})+\sim \\
50 \mathrm{vol} \%(1 \mathrm{M} \\
\mathrm{NaOH})\end{array}$ & 18 & 45 \\
\hline \multirow[b]{2}{*}{2 \#119 } & \multirow{2}{*}{$\begin{array}{l}\text { Sequential approach: } \\
\text { First etching from both sides without } \mathrm{Li}^{+} \text {and } \mathrm{F}^{-} \text {pres- } \\
\text { ence; etching stopped after breakthrough; thereafter in- } \\
\text { sertion of } \mathrm{Li}^{+} \text {and } \mathrm{F}^{-} \text {solutions without etchant on both } \\
\text { sides: Smooth Ohmic ionic passage through tracks with } \\
\text { relatively constant current for days; possibly transient } \\
\text { LiF membrane formation }\end{array}$} & $\begin{array}{c}\text { First: } \\
9 \mathrm{M} \mathrm{NaOH}\end{array}$ & $\begin{array}{c}\text { First: } \\
9 \mathrm{M} \mathrm{NaOH}\end{array}$ & 12 & 25 \\
\hline & & $\begin{array}{l}\text { Second: } \\
10 \mathrm{M} \mathrm{LiCl}\end{array}$ & $\begin{array}{l}\text { Second: } \\
1 \mathrm{M} \mathrm{NaF}\end{array}$ & 80 & 25 \\
\hline $3 \# 120$ & $\begin{array}{c}\text { Simultaneous approach: } \\
\text { Simultaneous etchant attack from both sides and } \mathrm{Li}^{+} / \\
\mathrm{F}^{-} \text {interaction. Smooth capacitive currents, no membrane } \\
\text { formation. }\end{array}$ & $\begin{array}{l}\text { High } \mathrm{Li} \text { concentr } \\
\sim 50 \mathrm{vol} \%(10 \mathrm{M} \\
\mathrm{LiCl})+\sim 50 \mathrm{vol} \% \\
\quad(5 \mathrm{M} \mathrm{NaOH})\end{array}$ & $\begin{array}{l}\sim 50 \mathrm{vol} \%(1 \mathrm{M} \\
\quad \mathrm{NaF}) \\
+\quad \sim 50 \mathrm{vol} \% \\
(1 \mathrm{M} \mathrm{NaOH})\end{array}$ & $\sim 100$ & 35 \\
\hline $4 \# 122$ & $\begin{array}{l}\text { Simultaneous approach: } \\
\text { Etching from both sides in the presence of } \mathrm{Li}^{+} \text {and } \mathrm{F}^{-} \\
\text {ions. Very low, constant Ohmic conductance, Arrhenius- } \\
\text { type temperature correlation. LiF precipitation within } \\
\text { latent tracks supposed; no LiF membranes found. }\end{array}$ & $\begin{array}{c}\sim 5 \mathrm{vol} \%(10 \mathrm{M} \\
\mathrm{LiCl})+95 \mathrm{vol} \% \\
(9 \mathrm{M} \mathrm{NaOH})\end{array}$ & $\begin{array}{l}\sim 50 \mathrm{vol} \% \\
(1 \mathrm{M} \mathrm{NaF})+ \\
\sim 50 \mathrm{vol} \%(1 \mathrm{M} \\
\quad \mathrm{NaOH})\end{array}$ & 30 & $35-85$ \\
\hline
\end{tabular}

obtain the total Li distribution across the whole foil with sufficiently good depth resolution. The spectra were evaluated off-line using the Libor code [7].

\section{Results and Discussion}

\section{Results of Electrical Measurements and ITS}

The parameters that determine the behaviour of the coupled chemical reactions are essentially the type and concentration of the liquids applied on both foil sides, the temperature and the voltage applied across the foil. According to earlier work based on current/voltage spectroscopy (see e.g. Figure. 7 in Ref. [3]), the best membrane formation should occur at $9 \mathrm{M} \mathrm{NaOH}$ concentration $\left(50 \mathrm{vol} \%\right.$ etchant in mixture with $\mathrm{LiCl}$ or $\mathrm{NaF}$ solution), $45^{\circ} \mathrm{C}$ etching temperature and 1 Vpeak-peak applied test voltage. For different configurations (e.g., $1 \mathrm{M} \mathrm{NaOH}, 20^{\circ} \mathrm{C}$ etching temperature, etc.), hardly any hint for membrane formation was derived from the current/voltage $(\mathrm{I} / \mathrm{U})$ protocols.

Also the ITS examinations reconfirmed the trend as seen in the I/U studies. Membrane formation occurs more frequently for short pre-CCR etching times than for longer ones, but nevertheless is quite rare $(\sim 1 \%)$. The reason for the sample deterioration with CCR etching time comes from the lack of possibilities of the precipitates to anchor firmly on the etched track walls, due to nanofluidic properties of the system. With longer pre-CCR etching times the previously smooth currents become spiky and pre- cipitates are ejected from the tracks, due to the onset of turbulent flow in the tracks. In general sequential experiments always allow the formation of membrane-containing etched tracks, whereas the simultaneously coupled chemical reaction arrangements hardly yield any. Depending on the experimental parameters, one either encounters here rather complete track blockage yielding permanent capacitive responses, or nanofluidic Ohmic-type currents.

All NDP measurements presented here were taken from the moment on when the I/U recordings (as presented in [3]) were stopped.

\section{Sequential Approach, foil Treatment before Breakthrough}

For the first sample (\#123), first fast etching took place from both sides with $9 \mathrm{M} \mathrm{NaOH}$ at ambient temperature without the presence of $\mathrm{Li}^{+}$and $\mathrm{F}^{-}$ions. The etching was stopped before breakthrough between both track sides occurs, and both foil parts were carefully cleaned with deionized water. Thereafter etching continued at $45^{\circ} \mathrm{C}$ from the Cis side with a $\{\sim 50 \mathrm{vol} \%(1 \mathrm{M} \mathrm{NaF})+\sim$ $50 \mathrm{vol} \%(1 \mathrm{M} \mathrm{NaOH})\}$ solution, whereas the Trans side was filled with $10 \mathrm{M} \mathrm{LiCl}$ solution only. The track breakthrough occurred during this second etching phase in pronounced steps until a stable Ohmic current was obtained for some time, followed again by stepwise strong current drops and rises until it remained constant for days. The intermediate current drops and the corresponding ITS measurements indicate the emergence of $\sim 0.5$ to $2 \mu \mathrm{m}$ thick $\mathrm{LiF}$ membranes in the etched tracks and thus reconfirm, in prin- 
ciple, our membrane formation model [1-3].

It was assumed that during the time of etchant breakthrough, when the emerging nanopore radii were still near-zero so that nanofluidic flow across the tracks was not yet properly established, the formed LiF nanocrystals still were in contact with the track walls and therefore could anchor there, thus forming membranes and blocking the subsequent current passage. Following our previous explanation [1], the later transient current rises would then stem from the different repair steps necessary to adjust the membrane size to the increasing track radii upon continuing track wall etching. Once the membrane's stability is no longer given due to excessive etched track size, they will break permanently and enable smooth nanofluidic ionic passage with little interaction, so that large amounts of $\mathrm{LiF}$ precipitates are formed at the track exits and accumulate in both compartments of the reaction vessel.

Figure 1a shows that the NDP spectra of this sample after washing (so that only LiF precipitates remain), as measured from both the Cis- and Trans-sides of the sample (corresponding to the directions towards or opposite to the diode direction in the $\mathrm{I} / \mathrm{U}$ measurements, respectively), are rather similar to each other. When evaluated and superposed (Figure 1b), they show two big surface peaks with exponentially decreasing concentration distributions on both foil surfaces. Unfortunately not any central LiF concentration enrichment showed up in the tracks as was expected from the membrane formation. This could signify on the one hand, that membranes might not be formed in all tracks, and on the other hand, that the overall amount of LiF precipitated as membranes in the narrow etched tracks is too small to be detected by NDP. A decision between these two alternatives is not easily possible. Anyhow, both the low Li concentrations in the track centres and the maxima on both foil sides verify our previous expectation of a rather smooth laminar interaction-free nanofluidic ionic passage through the etched tracks and an increased LiF precipitation only near the track exits, where larger track radii and the transition to the open vessel enable the onset of turbulent flow with $\mathrm{LiF}$ formation.

As long as the etched track radii are still below the thickness of the electric double layer (ELD), the negatively charged F- counter-ion transport through the etched track centres is still efficiently blocked, whereas the positive $\mathrm{Li}^{+}$ions may always pass closely to the negatively charged etched track walls to the opposite track side. This is why an accumulation of $\mathrm{Li}^{+}$concentration (and hence also LiF precipitation) is favoured on the opposite (Trans) foil side. Only when continuing etching lets the track radii exceed the ELD thickness, remarkable counter-ion transport will also set in. This explains why the NDP measurement yields a twice that high $\mathrm{Li}$ abundance on the $\mathrm{NaF}$ side (Trans side) as compared with the $\mathrm{LiCl}$ (Cis) side. (The observed difference in Li surface concentrations cannot be attributed to asymmetries of the etched track geometry as the NDP measurements in both directions yield more or less identical spectra, Figure 1a).

\section{Sequential Approach, foil Treatment After Breakthrough}

Also in this case the treatment of the given sample (\#119) was performed by a sequential approach; however, for comparison with the above experiment, in this case we added both the $\mathrm{Li}^{+}$and $\mathrm{F}^{-}$ions only after the breakthrough of the first etching stage. In this instance, no more etchant was added in the second stage. The observations of the corresponding I/U measuring protocol (Figure 10 in [3]) showed that during the initial etching phase some background currents emerged due to chemical potential differences, which subsequently gradually vanished. The abrupt onset of Ohmic currents was preceded by some spikes. After a period of irregular fluctuations, finally the Ohmic current became constant. This was tentatively explained by assuming a short-living transient LiF membrane formation in the initial phase, and by purely smooth nanofluidic ionic passage with little interaction at a later stage. Large amounts of $\mathrm{LiF}$ precipitations accumulated in both compartments outside of the etched ion tracks. The ITS spectra were similar to the above case. Only few etched tracks with $\sim 0.2$ to $\sim 0.6 \mu \mathrm{m}$ thick embedded $\mathrm{LiF}$ membranes were found. It is assumed that these membranes still stem from the very first moments of the insertion of $\mathrm{Li}^{+}$and $\mathrm{F}^{-}$ions into the pre-etched tracks and have blocked further accumulation of $\mathrm{LiF}$ in these pores since then. Again, it seems that the survival of such membranes is a rare case. In general the etched tracks are completely filled with $\mathrm{LiF}$ and the samples show strong statistically varying surface precipitations.

The fact that the ion tracks were already well-etched beyond the breakthrough condition, with radii probably exceeding the Debye layer thickness, signifies that both the $\mathrm{Li}^{+}$and $\mathrm{F}^{-}$ions are capable

Figure 1. a) NDP, raw spectra taken from Cis- (“outwards diode") and Trans- ("towards diode") sample sides. The energy spectra stemming from the tritons of the ${ }^{6} \mathrm{Li}(\mathrm{n}, \alpha)^{3} \mathrm{H}$ reaction are denoted by: $\mathrm{T}$, those stemming from the $\alpha$ particles are denoted by: $\alpha$. b) The overlap of the individually measured Li depth distributions of both foil sides yields the presented overall $\mathrm{Li}$ concentration distribution in the sample \#123, which is exponentially decaying on both sides. Note that the concentration scales are arbitrary.
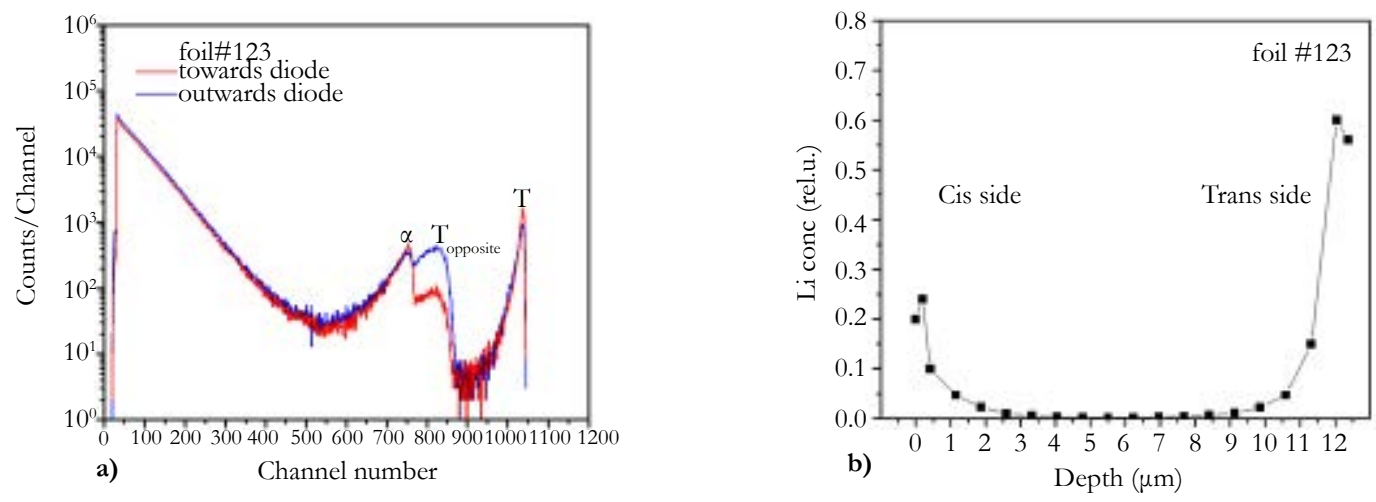

D Fink, J Vacik, V Hnatowicz, G Muñoz H, H García Arellano, et al., (2016) Coupled Chemical Reactions in Dynamic Nanometric Confinement: VI. Neutron Depth Profiling Studies 
of moving to the counter-ion side during this CCR phase, and hence produce roughly equal amounts of $\mathrm{LiF}$ precipitation yields on both surfaces. Therefore the NDP measurements (Figure 2) show Li depth profiles which have somewhat similar heights on both sides of the polymer foil. As the nanofluidic mechanism largely prevents the $\mathrm{LiF}$ precipitate anchorage on the ion track walls, the overall measured surface Li concentration is less than in the previous cases. Consequently, the smooth nanofluidic counter-directed flow of both ions and counter-ions within the etched tracks with little interaction between them moves most ions out of the tracks so that less $\mathrm{LiF}$ precipitate is found within the ion tracks.

\section{Simultaneous Approach, Capacitive Behaviour, Track Blocking}

The next sample (\#120) falls into the category of high-fluence $\left(80 \mathrm{MeV} \mathrm{Kr}, 5 \times 10^{7} \mathrm{~cm}^{-2}\right.$ ) irradiated targets as distinguished in the previous work [3]. The etchant attack was performed from both sides in the presence of high concentrations of $\mathrm{Li}^{+}$and $\mathrm{F}^{-}$ions. According to our previous understanding [3], these ions (preferentially $\mathrm{F}^{-}$) migrated rapidly along the latent tracks towards the opposite foil sides where they underwent the formation of impermeable LiF surface layers that - already after less than 5 min - seem to block both the further track etching and the track conductivity, thus leading to purely capacitive current signals that persisted for days. However, in contrast to these conclusions, ITS of this sample showed that remarkable bulk foil etching did take place. This signifies that the $\mathrm{LiF}$ layer formed on the foil surfaces did not prevent the $\mathrm{OH}^{-}$ions from etching the polymer foil, though it prevents Ohmic currents to flow across the measuring system. The latter finding is understood by taking into account that both the charge carriers $\mathrm{Li}^{+}$and $\mathrm{F}^{-}$transported across the foil annihilate at the opposite surfaces by formation of neutral LiF. No LiF membranes were found by ITS, but all ion tracks are filled with LiF. Strong and rather homogeneous LiF precipitations were found both on the sample surface and in the vessel.

Additionally to the above findings, the corresponding NDP measurements, Figure 3, indicate strong differences between both foil sides insofar as there is much more LiF precipitation on the Cisside (left side in Figure 3b) than on the Trans side. As the Cis-side was that foil side which was in contact with the $\mathrm{LiCl} / \mathrm{NaOH}$ so- lution, it indicates (as was already derived from the initial visible inspection) that preferentially rapid $\mathrm{F}^{-}$ion migration along the latent tracks took place from the Trans side towards the Cis side, where both the $\mathrm{Li}^{+}$and $\mathrm{F}^{-}$components formed LiF. After subsequent sample washing with water only this immobile LiF fraction (but not the $\mathrm{Li}^{+}$ions from the original solution) survived and was detected here by NDP.

Apparently the $\mathrm{Li}^{+}$ions diffuse much more slowly within the polymer along the latent tracks. The reason for this may be found in the very large hydrate shells around $\mathrm{Li}^{+}$ions in solution that dramatically inhibits their migration, as compared with the smaller $\mathrm{F}^{-}$ions. Upon their bulk migration the $\mathrm{Li}^{+}$ions encounter $\mathrm{F}^{-}$ions within the latent tracks and become trapped by the formation of immobile LiF. Such a diffusion/trapping process is known to lead to exponentially decaying concentration profiles, as it was found indeed here (Figure 3b).

\section{Simultaneous Approach, Ohmic Behaviour, LiF Emission}

This sample (\#122) falls into the category of low-fluence (80 $\mathrm{MeV} \mathrm{Kr}, 45 \times 10^{6} \mathrm{~cm}^{-2}$ ) irradiated targets as distinguished in the previous work [3]. The etchant attack was performed from both sides in the presence of low concentrations of $\mathrm{Li}^{+}$and $\mathrm{F}^{-}$ions. $\mathrm{I} / \mathrm{U}$ measurements reveal that the sample shows a very low, constant Ohmic conductance that follows an Arrhenius-type temperature correlation at least in the interval from 35 to $85^{\circ} \mathrm{C}$. The tracks exhibit some limited $\mathrm{Li}^{+}$and $\mathrm{F}^{-}$permeation due to etching combined with LiF precipitation.

ITS measurements on this sample also reveal that in this case membranes are never formed but that all etched tracks are completely filled with LiF. Also many strongly inhomogeneous LiF surface precipitations are found here.

The corresponding NDP measurements show that there are very few differences between transmission from one foil side and the other, which indicates some similarities of the internal sample geometry and kinetics on both sides, Figure 4. The Li concentrations on both foil surfaces are strong, with exponential-type concentration distributions towards the sample's interior. Due to both a higher etchant concentration, lower $\mathrm{Li}$ concentration and higher sample temperature as compared with the previous case,

Figure 2. a) NDP, raw spectra taken from Cis- (“outwards diode") and Trans- ("towards diode”) sample sides. The parts of the energy spectra stemming from the tritons of the ${ }^{6} \mathrm{Li}(\mathrm{n}, \alpha)^{3} \mathrm{H}$ reaction are denoted by: $\mathrm{T}$, those stemming from the $\alpha$ particles are denoted by: $\alpha$. b) The overlap of the $\mathrm{Li}$ depth distributions of both foil sides yields the overall Li concentration distribution in the sample \#119, which is again exponentially decaying on both sides. Note that the concentration scales are arbitrary.
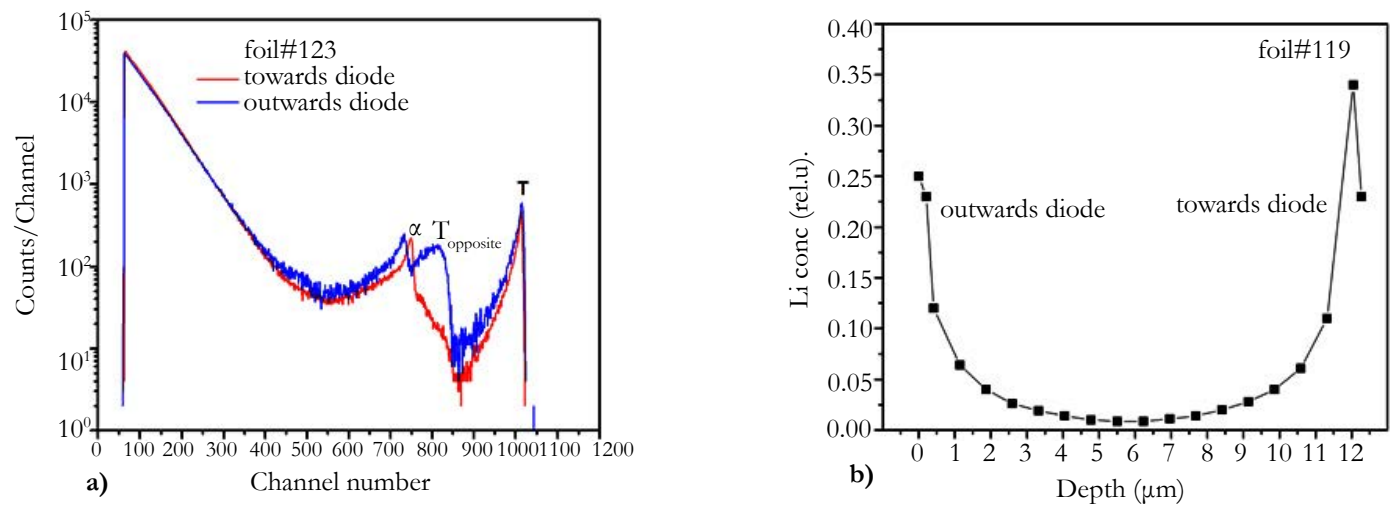

D Fink, J Vacik, V Hnatowicz, G Muñoz H, H García Arellano, et al., (2016) Coupled Chemical Reactions in Dynamic Nanometric Confinement: VI. Neutron Depth Profiling Studies 
Figure 3. a) NDP raw data of sample \#120, taken from Cis- (“outwards diode") and Trans- (“towards diode") sample sides.

The energy spectra stemming from the tritons of the ${ }^{6} \mathrm{Li}(\mathrm{n}, \alpha)^{3} \mathrm{H}$ reaction are denoted by: T, those stemming from the $\alpha$ particles are denoted by: $\alpha$. b) Exponential Li concentration distribution in foil \#120, as determined from the overlap of two NDP measurements from both foil sides. Note that the concentration scales are arbitrary.
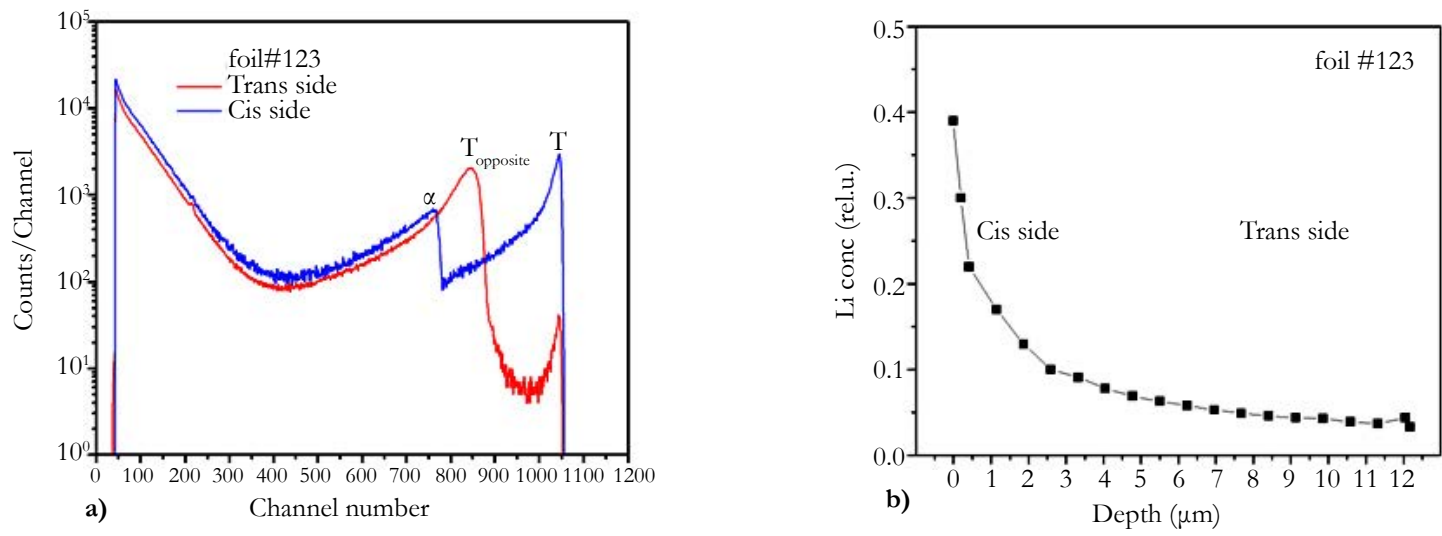

Figure 4. a) NDP, raw spectra taken from Cis- (“outwards diode") and Trans- (“towards diode") sample sides. The energy spectra stemming from the tritons of the ${ }^{6} \mathrm{Li}(\mathrm{n}, \alpha)^{3} \mathrm{H}$ reaction are denoted by: $\mathrm{T}$, those stemming from the $\alpha$ particles are denoted by: $\alpha$. b) Exponentially decaying Li concentration distributions on both sides of the ion-irradiated foil \#122, as determined from the overlap of two NDP measurements from both foil sides. Note that the concentration scales are arbitrary.

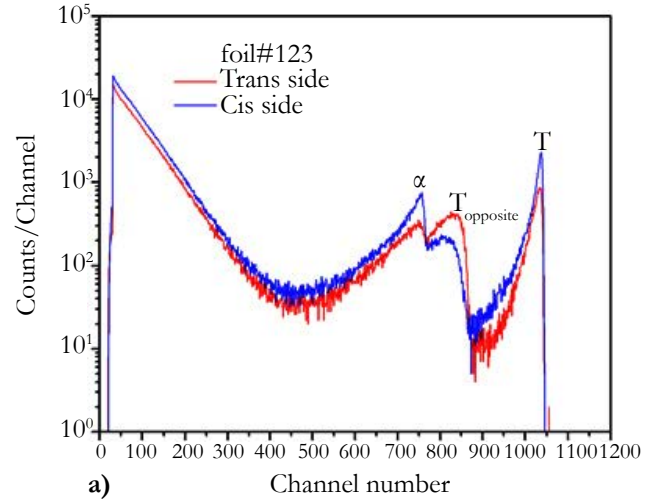

both simultaneous track etching and higher Li diffusion along tracks occur, hence the $\mathrm{Li}$ penetration is deeper and hence LiF formation is also found on the opposite foil side. The emerging nanofluidic effect lets both ions and counter-ions pass through the whole foil without reacting inside the tracks. They rather react at the track exits towards the formation of strong LiF precipitations on both foil sides. As the mechanism underlying nanofluidic ion transport is a diffusive one, the previous finding of an Arrhenius behaviour in this case is evident.

These findings support the model of a limited permeation of both ions towards both foil sides, and it also indicates the validity of a nanofluidic description, thus leading to somewhat similar LiF precipitation on both surfaces: as compared with a difference of a factor $\sim 50$ or so for the previous sample \#120, the LiF precipitation on the $\mathrm{LiCl}$ side exceeds here that one on the $\mathrm{NaF}$ side here by only a factor 2 to 3 .

\section{NDP Examinations of CCR-Modified Tracks with LiF Membranes: Summary and Outlook}

Due to the special reaction type in this case, the two chemical reactions (track etching and LiF precipitation formation) can be either performed sequentially or simultaneously (i.e., coupled with each other). Whereas ITS measurements of all sequential experi-

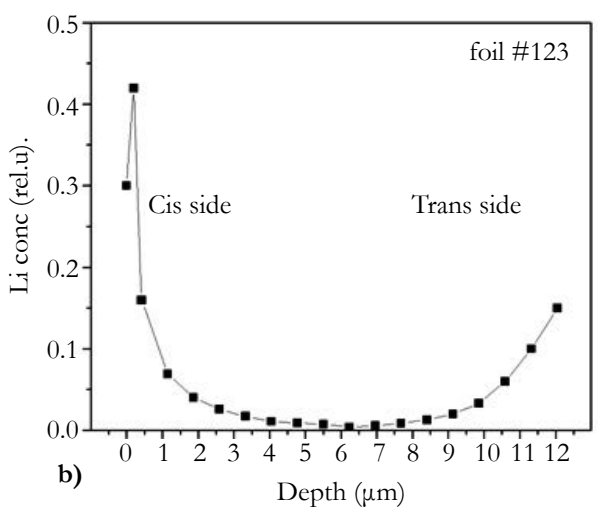

ments indicated the formation of at least some (though very few) membrane-containing etched track, none of the coupled chemical reaction arrangements yielded any. Whereas the results of the experiments for sequential LiF-formation strongly resemble those with $\mathrm{Ag}_{2} \mathrm{O}$ precipitations (see [5]), the results of the simultaneously coupled chemical reaction (CCR) experiments are strikingly different. Here one either encounters rather complete track blockage yielding permanent capacitive responses, or nanofluidic Ohmic-type currents, depending on the experimental parameters.

In this work on coupled \{track etching/LiF precipitation experiments, we did not yet examine the influence of the post-etching time for the sequential etching arrangements. It is, however, expected that - similarly as was shown for $\mathrm{Ag}_{2} \mathrm{O}$ formation [5] - also here the fraction of tracks which contains membranes will increase with decreasing post-etching times. This should be examined in a future work.

Consider the differences in $\mathrm{Li}^{+}$and $\mathrm{F}^{-}$mobilities in the polymeric tracks: As long as the ionic migration takes place in latent ion tracks, the $\mathrm{F}^{-}$diffusion seems to exceed the $\mathrm{Li}^{+}$diffusion by far (probably due to the large hydrate shells around $\mathrm{Li}^{+}$ions that disables their easy bulk migration). In this case, LiF precipitations occur preferentially on the Cis-side of the $\mathrm{Li}^{+}$ion compartment. When the tracks are marginally etched (so that the radii of the emerging nanopores are roughly comparable with the thickness 
of the ELD), then the nanofluidic $\mathrm{Li}^{+}$ion transport is favoured and the $\mathrm{F}^{-}$transport suppressed due to the much larger crosssectional track area available for $\mathrm{Li}^{+}$transport than for $\mathrm{F}^{-}$. However with prolonged track etching, this cross-sectional area ratio changes so that $\mathrm{F}^{-}$counter-ion transport becomes dominant. As the LiF precipitates are swept out of the tracks by friction along the neighbouring liquid, we will find finally dominant LiF precipitation at the Cis-side.

\section{Acknowledgement}

This project was supported by the Grant Agency of the Czech Republic (P108-12G-108). D.F. is grateful to the Universidad Autónoma Metropolitana-Cuajimalpa, Mexico City, for the guest professorship in the frame of the Cathedra "Roberto Quintero Ramírez" and to both the Ben Gurion University of the Negev, Beer Sheva and the Nuclear Physics Institute, Rez, for providing travel support. We are further obliged to Dr. P. Apel from JNRI Dubna, Russia for providing us with the ion-irradiated polymer foils. L.A. would like to acknowledge the Edmond J. Safra Center for the Design and Engineering of Functional Biopolymers in BGU.

\section{References}

[1]. G Muñoz H, SACruz, D Fink, L Alfonta, Y Mandabi, et al., (2013) I. Coupled chemical reactions in dynamic nanometric confinement: $\mathrm{Ag}_{2} \mathrm{O}$ formation during ion track etching. Radiation Eff Def Matter. 168(9): 675-695.

[2]. D Fink, G Muñoz H, H García Arellano, WR Fahrner, K Hoppe, et al., (2014) Coupled chemical reactions in dynamic nanometric confinement: II. Preparation conditions for $\mathrm{Ag}_{2} \mathrm{O}$ membranes within etched tracks. "Technologies for smart sensors and sensor fusion", CRC Press, Boca Raton, Florida, 451-462.

[3]. D Fink, G Muńoz H, NL Ruiz, SA Cruz, J Vacik, et al., (2014) Coupled chemical reactions in dynamic nanometric confinement: $\mathrm{V}$. The influence of $\mathrm{Li}+$ and $\mathrm{F}$ - ions on etching of nuclear tracks in polymers. Radiation Eff Def Matter. 169(5): 1-22.

[4]. P Abgrall, Nam-Trung Nguyen (2009) Nanofluidics. Artech House, Norwood, MA.

[5]. D Fink, J Vacik, V Hnatowicz, G Muñoz H, H García Arellano, et al., (2015) Coupled chemical reactions in dynamic nanometric confinement: IV. Ion transmission spectrometric analysis of nanofluidic behaviour and membrane formation during track etching in polymers. Radiat Eff Def Matter. 170(3): 155-174.

[6]. D Fink (1996) Neutron Depth Profiling. Report HMI-B539, Hahn-Meitner-Institute Berlin.

[7]. V Hnatowicz, J Vacik, D Fink (2010) Deconvolution of charged particle spectra from neutron depth profiling using Simplex method. Rev Sci Instrum. 81: 073906 . 